\title{
Significance Testing Needs a Taxonomy
}

Bradley, Michael T.; Brand, Andrew

\section{Psychological Reports}

\author{
DOI: \\ $10.1177 / 0033294116662659$
}

Published: 01/10/2016

Peer reviewed version

Cyswllt i'r cyhoeddiad / Link to publication

Dyfyniad o'r fersiwn a gyhoeddwyd / Citation for published version (APA):

Bradley, M. T., \& Brand, A. (2016). Significance Testing Needs a Taxonomy: Or How the Fisher, Neyman-Pearson Controversy Resulted in the Inferential Tail Wagging the Measurement Dog. Psychological Reports, 119(2), 487-504. https://doi.org/10.1177/0033294116662659

\footnotetext{
Hawliau Cyffredinol / General rights

Copyright and moral rights for the publications made accessible in the public portal are retained by the authors and/or other copyright owners and it is a condition of accessing publications that users recognise and abide by the legal requirements associated with these rights.

- Users may download and print one copy of any publication from the public portal for the purpose of private study or research.

- You may not further distribute the material or use it for any profit-making activity or commercial gain

- You may freely distribute the URL identifying the publication in the public portal ?
}

Take down policy

If you believe that this document breaches copyright please contact us providing details, and we will remove access to the work immediately and investigate your claim. 
RUNNING HEAD: TAXONOMY RESOLVES THE FISHER, NEYMAN PEARSON CONTROVERSY

Significance testing needs a taxonomy: or how the Fisher, Neyman-Pearson controversy resulted in the inferential tail wagging the measurement dog. 


\begin{abstract}
Accurate measurement and a cutoff probability with inferential statistics are not wholly compatible. Fisher understood this when he developed the $F$ test to deal with measurement variability and to make judgments on manipulations that may be worth further study. Neyman and Pearson focused on modeled distributions whose parameters were highly determined and concluded that inferential judgments following an $F$ test could be made with accuracy because the distribution parameters were determined. Neyman and Pearson's approach in the application of statistical analyses using alpha and beta error rates has played a dominant role guiding inferential judgments, appropriately in highly determined situations and inappropriately in scientific exploration. Fisher tried to explain the different situations, but, in part due to some obscure wording, generated a long standing dispute that currently has left the importance of Fisher's $p<.05$ criteria not fully understood and a general endorsement of the Neyman and Pearson error rate approach. Problems were compounded with power calculations based on effect sizes following significant results entering into exploratory science. To understand in a practical sense when each approach should be used, a dimension reflecting varying levels of certainty or knowledge of population distributions is presented. The dimension provides a taxonomy of statistical situations and appropriate approaches by delineating four Zones that represent how well the underlying population of interest is defined ranging from exploratory situations to highly determined populations.
\end{abstract}


Significance testing needs taxonomy: or how the Fisher, Neyman-Pearson controversy resulted in the inferential tail wagging the measurement dog?

A hallmark of science is measurement and measurement is characterized by accuracy and precision. Probability estimates involve greater or lesser degrees of uncertainty, but statistical procedures associated with inferential statistics for some characterize the scientific approach in psychology (Babbie, 2001, p 452). These procedures have been extensively developed and are influential in reporting the results of applied and behavioral studies. Great attention has been paid to the correct mechanics of calculation, but somehow questions about the meaning of significant results following of inferential tests while discussed by many (for examples, Cohen, 1994, Rosenthal, 1979, Sterling, 1959) have taken some time to have an impact. That impact has possibly arrived with the American Statistical Association's statements on p values (Wasserstein and Lazar, 2016). Such papers, at the least, prime the field to ascertain how well probability results reflect measurement accuracy.

Fisher recognized that results following an inferential test are the lowest level of scientific inference (Box, 1978, pages 447-448). He distinguished different situations for the judgment of results: 1) research involving exploratory scientific endeavors, and 2) statistics associated with production processes and quality control. Exploratory research involves inductive reasoning, and going from "consequences to causes" page 3, Fisher, 1942). Box (1978, p 448) points out that statisticians looking for a single general solution and favoring deductive reasoning were not satisfied with Fisher's approach. Fisher offered a variety of inferential approaches appropriate to some but not all problems. This was evident in the way 
that he classed the work of Neyman \& Pearson (1933). He thought their routine mathematical deductive way of judging statistical results was appropriate and valuable for quality control, but their approach was not legitimate when it spread into exploratory science (Fisher, 1955, p 69). Several authors (see a review Perezgonzales, 2014) state that researchers follow a "hybridization" of the approaches especially regarding significance levels with some aspects of inferential procedures being of limited value and other aspects being impediments to progress in science (Branch, 2014). Fisher (1942, p 8) was insistent that the value of inferential statistics was tied to the logical assumptions of the context of data collection.

In each specific area where inferential statistics are used, a particular population distribution exists. In manufacturing or quality control situations the population distribution may be limited and fully defined as the result of the normal processes of the factory. For example, some index, such as product weight, size, color, etc. would be defined by a mean and standard deviation. Differences from the population distribution can be readily specified. Inspired by Neyman and Pearson (1933), who set up a model sampling regime specifying two types of error: Type 1 or alpha error and Type 2 or beta error, Wald and Abraham (1945) developed tests appropriate for manufacturing. Type 1 error, set at $\alpha=.05$, represents the probability of being wrong if it is concluded that a deviation larger than this threshold is considered not representative of the population distribution. Type 2 error, usually set at $\beta=.10$ or .20 , is the probability of concluding that there is no difference when one actually exists. The correctness of these error rates are conceptualized as "long-run" probabilities borne out through repeated testing afforded in sampling for quality control (Halprin \& Stam, 2006). 
Fisher (1973) points out that the manufacturing situation contrasts with scientific exploration. He states "... to identify a test of significance as used in the natural sciences with a test for acceptance, one of the deepest dissimilarities lies in the population ...used for making statements of probability.(p 81)". The true population distribution in science, at least at the outset of exploration, is not known, and this contrasts with a well-described Neyman and Pearson type of population. As a consequence with the exploratory population, one cannot with any certainty suggest error rates in terms of alpha (Type 1) and beta (Type 2) probabilities. In other words, a researcher divides a sample of the unknown larger population into groups some or one of which is/are treated, and in that context the most legitimate judgment is categorical, depending upon the probability level, in that groups are either like or unlike each other. Usually this is assessed by mean differences, although other distribution qualities could be relevant (Bradley, Brand \& MacNeill, 2012, 2013; Tukey, 1977). That judgement for Fisher would be against the null hypothesis if the probability was $p \leq .05$. That such a treatment might work in another circumstance is in all likelihood reasonable and all the more reasonable as the probability gets smaller. It does not, however, yield something as definite as an error rate. If one does not know the true population distribution, it is misleading to set error rates based on exploratory studies and imply they have validity. Fisher made it clear that "... tests of significance which make a dichotomy between hypotheses that are discredited by the data and those that are not..." are, as mentioned earlier, the lowest level of scientific inference (Box, 1978, pages 447-448).

All samples of interest are a subset of the population distribution, and of course in that sense the sample estimates approximate the parameters of population distribution. As Fisher put it, sample values provide estimates of population parameters (Fisher, 1973, p 54).The sample, however, (for example a particular University class) that any researcher may select could be 
substantially different from the more general population distribution of interest to the researcher to begin with. For example, Sharpe and Poets (2015) reviewed the literature noting the paucity of males in psychology experiments. Neyman and Pearson (1933) solved the sample - population difference problem by making their population distribution the very one from which they were sampling and resampling (Fisher, 1955). Thus, they could specify errors from their known population. Fisher (1973, p 81) labeled them acceptance procedures for a product.

Fisher (1973, p 54-55) had, early in his career, introduced a fiducial approach to be appropriate when no other information was available. This approach, borrowed from astronomy and land surveying, establishes a reference point from which measurements are made (Stigler, 1986, p 101). The insight was that probability statements could be calculated within the data set or sample at hand in just the same way that a path or acre could be measured off a fiducial point in land survey. The limits, however, are two. In land survey the scale of measurement is of the highest order, ratio scale (Stevens, 1946), whereas for Fisher the measurement in $S D$ units is only relevant to the sample, and the categorization of mean differences is dependent on probabilities calculated on standard error (se) units. Thus the $S D$ as a representative estimate is only as good as the sample, and the se essentially supplies an increment that is the equivalent to a rubber ruler that varies with the $n$. Thus, Fisher (1935) could only make categorical statements such as the treated half of the sample is probably not like the untreated half of the sample. His probability statements based on estimates from his initial sample were not exact to the population parameters, but were predictive of future tests on samples of the same topic. In fact, Fisher (1973) stated that "...the only populations that can be referred to in a test of significance have no objective reality, being the product of the statistician's imagination...(p 81)". In other words, the 
researcher has no a priori knowledge of the center and shape of the imagined distribution. What Fisher can conclude following a significance test "...of a small $P$-value is that something very unlikely has happened or the null is false ..." (Keuzenkamp and Magnus, 1995, p 18). Neyman and Pearson believed they had defined a stable population distribution through repeated sampling, whereas Fisher estimated the parameters of a fundamentally undefined population distribution from the sample.

The argument is that the Neyman and Pearson model does not work in science. In science the population parameters are not known, and the sample values are estimates of the population imagined by the researcher. For Fisher (1973) differentiating between two groups in a sample was valuable, but the most primitive form of measurement ( $\mathrm{p} 46$...it is more primitive, or elemental...). It has the status of furthering the inductive reasoning process but is not definitive when trying to generalize to all members of a class or population. To make the difference less abstract, consider the research concerns of Fisher such as manure on crops, or the deterioration of food in storage (Fisher, 1973, p 79). With data, he could not say a program of manure would be exactly as successful in a following year, but depending on the probability levels, he could make a reasoned statement that this program is worth doing the following year. With more research he could decide to look at different varieties of a crop, but until the population is completely defined only probabilities, not error rates, can be reported. Fisher (1973, 112-113) noted that a research scientist was dealing with inductive reasoning “...to improve our understanding of the systems from which these observations are drawn". Fisher (1955, p 69) repeated this in an article on scientific induction as a major advantage to his and Gosset's approach (Student, 1908) in the introductory paragraph. Once the population distribution is fully identified and specified then it is possible to apply the deductive approach 
favored by Neyman and Pearson (1933) with error rates (5\% chance of Type 1 and a 10\% or $20 \%$ chance of Type 2 error). Until then, however, the control sample is unspecified in its approximation to the population distribution, and a specification of Neyman and Pearson errors at this stage would refer only to the difference between the control and manipulated sample(s). Error rates imply accuracy and precision that is unwarranted if someone applied the results to another group from a different but as yet unknown sector of the population. With Fisher's (1973, p128) recommendation the report is of a result improbable by chance exactly between the groups sampled and tested and in the early stages of research no more than that. Another researcher may get a smaller or larger $\mathrm{p}$, and over replications the extent of the difference and the likelihood of that difference becomes known, maybe to the point with many samples where the Neyman and Pearson's approach applies. By then, Fisher (1973, p 80-81) would classify this as a use of statistics valuable as "acceptance procedures" but not for scientific exploration.

In general, Fisher (1973, pages 80-81) described the difference between situations (acceptance procedures) where an error approach referencing a known population distribution is appropriate and where a probability approach identifying a difference between two distributions is appropriate. This distinction anticipates a classification such that statistical approaches can be placed on a dimension. This dimension classifies broad areas in which a researcher could make a case for adopting particular form of judgment, error versus probability, appropriate for her/his investigation. It is argued that an approach that differentiates various areas under study resolves much of the controversy between Fisher and Neyman-Pearson. There are a variety of situations in which probabilities are appropriate, but the choice of judging the meaning of the resultant probabilities depends on what the researcher is examining. 
Fisher (1973, p 61) favored reporting exact $p$ values that were calculated from specific data sets being analyzed. He suggested distributions differed from each other when the probability was less than .10 or .05 and became more confident that the distributions differed with a lower $p$ value such as .01 with the provision that "...no scientific worker has a fixed level... (p 45)" for rejection. At higher levels of probability, he would simply withhold judgment regarding whether the experimental variable failed to have an effect since "the null hypothesis is never proved or established, but possibly disproved..." (Fisher, 1942, p 16). Neyman and Pearson (1933) saw Fisher's "no judgment possible" with regards to supporting the null as unnecessarily limited.

Neyman and Pearson (1933) did not advocate reporting exact probabilities. Rather, they suggested that that once the .05 level is crossed a decision to reject the null hypothesis will be erroneous only one time in twenty. "In making decisions (a) or (b) we shall sometimes be in error, for problems are rarely presented in such a form that we can discriminate with certainty between the true and the false hypothesis. These errors will be of two kinds: (I) we reject $\mathrm{Ho}$ when it is true, (II) we accept $H o$ when some alternative $H i$ is true. The problem before us is to consider how these errors may be. In a reciprocal fashion, if the beta error rate is low, .10 or less, then the acceptance of the null hypothesis through a failure to find significance would result in an error only 1 in ten times" (page 493).

In sum inferential statistics can be used either for monitoring or for research, but which depends upon whether the results are judged by error rates or probabilities. A researcher can reason from probability judgements as to whether there is enough evidence to pursue a phenomenon of potential interest and are not designed for accurate and precise measurement 
(Fisher, 1955, p 73). A small probability simply says that two or more samples differ to such a degree that one or more samples is/are not like the reference sample. Any particular effect size calculated to reflect differences between the distributions could be accurate, but in general the average calculation of a set of effect sizes following inferential statistics will not be accurate if power is less than an impossible $100 \%$ with the standard criterion of $\mathrm{p} \leq .05$ applied as a cutoff value. Accuracy is the closeness of a measured result to the true underlying value and to the degree that a significant result is inaccurate effect size measures will be inaccurate through exaggeration.

Precision or imprecision is a little more difficult to gauge. Underpowered studies, if published under .05 alpha constraints, inherently produce inaccurate estimates if effect sizes are calculated (for examples of discussion, Bakan 1966, Brand, Bradley, Best, and Stoica, 2008, Cohen, 1994, Gigerenzer, 1993, Kline, 2013). Even under the best of circumstances (90\% power, a normal distribution), Bradley and Brand (2016) showed with Monte Carlo results that the probability estimates from inferential statistics are $6 \%$ larger than the actual value tested. Further and pertinent to precision, these estimates would probabilistically be around the .05 level and could appear deceptively precise if the studies had approximately the same sample size. Of course, if there were a variety sample sizes in underpowered studies, the estimates would vary. Currently the publication manual of the American Psychological Association helps remedy this problem by suggesting the calculation of effect sizes and CIs associated with non-significant results (APA manual $6^{\text {th }}$ ed, 2010, p 33). There has been a definitive change as the $4^{\text {th }}$ edition only encouraged effect size reporting, and the $5^{\text {th }}$ edition indicted that a fuller understanding of the results would be conveyed if the effect sizes were reported (APA manual $4^{\text {th }} \& 5^{\text {th }}$ eds, 1999 , 2002). If the suggestions of the $6^{\text {th }}$ edition are followed, which include even non-significant 
effect size reporting, the $C I s$ would be more legitimate reflections of the set of effect sizes. If, however, the bias towards focusing on effect sizes calculated after significance remains, then the CIs for generally underpowered studies would be particularly distorted if the sample sizes in the set of studies varied substantially (Bradley \& Stoica, 2004, Brand \& Bradley, 2016). Of course once the probability screening criteria is dropped, it is no longer inference but becomes measurement.

Fisher saw inferential tests and particularly the $F$ test as useful but the most rudimentary of measurement tools (Box, 1978, pages 447-448). . They simply allowed the basic judgment of whether a new sample conformed to or deviated from the reference or null distribution. He and any researcher could state this in probability terms, as had been done previously by others using the term probable error (Cowles, \& Davis, 1982). Initially, he was fairly casual about the famous $p \leq .05$ level. In fact, he said that those who insisted on the .05 level did not know statistics: “... significance... is more primitive, or elemental than, and does not justify, any exact probability statement about the proposition (Fisher, 1973, p 46)." The quote is key to the controversy, because Neyman and Pearson (1933) were making exact statements. Fisher entertained a variety of potential significance levels depending upon the experimental circumstance, sample sizes, and distribution moments. He gave prominence to the .05 level, but it was mentioned in the context where .10 could be of interest depending on the circumstance. He did balance his thoughts by mentioning $p<.01$ as a hard criteria to meet and strongly suggestive of support for a phenomenon, and then came back to .05 as worthwhile to promote further investigation. Perhaps Fisher succinctly phrased it with “...no scientific worker has a fixed level of significance at which from year to year, and in all circumstances, he rejects hypotheses; he rather gives his mind to each particular case in the light of the evidence and his 
ideals." (Fisher, 1973, p 45). In specific regards to non-significance Fisher (1955) said, “The deviation is in the direction expected... and to this extent my suspicion has been confirmed...the data available....is not ... sufficient to demonstrate their reality.” p73). As an area is known then “... a properly designed experiment rarely fails to give this level of significance...” (Fisher 1926, p 504, original author emphasis). It is notable that Fisher wanted the exact level of $p$ reported for each replication attempt, assuming a somewhat consistent and reasonable sample size for each experiment, and by reasonable we mean a sample size likely to yield statistically significance results. Fisher's views are harmonious with current APA (2010) recommendations.

In 1935 Neyman orally presented a paper with Fisher in the audience meant to "....rectify the deficiencies of Fisher's thought" (Box, 1978, p263). Fisher (1955) said that Neyman "denies the existence of inductive reasoning" ( $\mathrm{p} 74$ ) and thought in terms of deductive reasoning. He with Pearson meant to sharpen the accuracy of Fisher's technique by considering situations where the populations of interest were limited and could be specified with exactitude. As mentioned Wald and Abrahamson (1945) applied the reasoning to quality control situations where inputs can be standardized and controlled. A context such as that comes pretty close to meeting scientific criteria for measurement. Thus, Neyman and Pearson's approach has been appropriately applied to production lines, because the production process determines with exactitude the input, and the physical or qualitative aspects of the final product can be specified in measureable terms (Lenhard, 2006). In addition, production processes involve reiteration or multiple opportunities for replication. With such knowledge, a sampling routine with probabilistic expectations of finding deviations from the expected product can be set up. The issue in this context is to discover a sample size that could in turn discover deviations with only a $5 \%$ chance that any particular deviation of that magnitude did not represent some problem with 
the production process and an $80 \%$ or $90 \%$ chance of discovering the deviation that reflected the problem. Of course, these values represent alpha or Type 1 error rates and the $10 \%$ or $20 \%$ (power, $100 \%-90 \%$ or $80 \%$ ) reflects the beta or Type 2 error rate. With several tests a number reflecting "normal" production processes deviations from normal could be derived such that a decision to 1) continue or 2) check the process could be made. A production process involves a well determined model with known properties. It is an appropriate application of technology. It is not the generation of new facts or "an intelligent attempt to understand the experimental situation.” (Fisher, 1955, p77, original author emphasis). The reason it is not "exploratory science" is that Neyman and Pearson's distribution is limited to a distribution that can be completely known. Importantly, that distribution does not pertain to all distributions of that type, because if, for example, a new manufacturing process were set up a new distribution associated with indices of the product would have to be explored.

The Neyman and Pearson approach of specification of Type 1 and Type 2 error rates can be done legitimately if virtually all aspects of the data gathering situation are determined. What Neyman and Pearson are really assuming is that a priori they know all about or enough about the reference distribution that they can, with small probabilities of error, suggest when a measured distribution deviates from or remains within the parameters of that distribution. In such circumstances, inferential statistics may become unnecessary and tolerance numbers derived from exact estimates of effect size may indicate the soundness of a process. For example, if a product should have a certain weight, a certain number of deviations from that weight may reflect a production problem. 
When the population distribution from which the sample is drawn are unknown it is not possible to specify population probabilities as error rates. Fisher (1973) put it clearly many times "...the evidence available concerns one population only, and tells us nothing more of the parent population that might lie behind it...(1973, p 124, for example)" The model of the reference distribution only emerges after the data has been collected, and it is only possible to infer if a difference between the reference distribution and a particular comparison distribution is improbable enough to make the pursuit of further investigation potentially worthwhile. David and Edwards (2001, p 188) also stated Fisher’s notion clearly “.... a probability statement about a parameter based on a fiducial distribution has the same inductive content as if the parameter had been drawn at random from a conventional probability distribution of identical form..." What makes the Neyman-Pearson testing situation so different is the assumption that the distribution sampled from is always the same known distribution, which is true in the context of product testing, but specifically not in exploratory situations. Fisher's problem was how to make “...inferences from the particular to general...” from variable data (Box, 1978, p 447). He was unsettled that advocates of the Neyman and Pearson approach did not seem to make a distinction between technical measurement and scientific exploration. If a distribution is defined beforehand "Nothing essentially new can be discovered..." (Fisher, 1973, p 112). This was unsettling because in his early surmise of his contribution Fisher (1922, p323) had noted "...the number and variety of the new results the method discloses...").

Fisher's choice of words to describe his points could result in problems. He used "imaginary" and "fiduciary". "Imaginary" referred to the underlying population that the researcher had in mind for generalization (Fisher, 1955, p71). A researcher can only sample from 
the population in mind with the particular portion of the population at hand. This leads to "fiduciary" probabilities or probabilities in reference to the sample being analyzed (Fisher, 1935). A researcher does not have an entire defined population. She/he has only a particular part of the population to sample from, in Fisher's case, a particular wheat field in England. The estimates from the sample serve as reference or fiducial points and may or may not adequately generalize to the "imagined" population (all wheat fields in England, the world?). An experiment is an instance to be generalized, but "The governing characteristic of inductive reasoning is that it is always used to arrive at statements of uncertainty... (Fisher 1973, p113). Fisher could agree that the generalization may be reasonable depending on a $p$ value following a manipulation, but Fisher would never agree that error probabilities had been legitimately specified with respect to the abstract population to which a researcher "imagined" the results pertained.

Fisher tried to explain the difference between his approach and that of Neyman and Pearson. $P$ values are not error rates, rather they indicate the probability that data after a treatment are like data from an untreated sample. The smaller the $p$ the more likely the treated sample is different than the untreated or null sample. Fisher $(1973, \mathrm{p} 81)$ made it clear that identifying significance testing in science with an acceptance test in production fails to recognize the deep dissimilarity “...in the population, or reference set, available for making statements of probability ...”. Fisher complained that Neyman and Pearson had been insulated from natural science (Fisher, 1955, p 70), and thus they failed to understand the tentative or exploratory nature of the few samples obtained in any given area. 
Perezgonzales (2014) is one of many who have noted that there is a danger in using a hybrid conception of inferential statistical testing. There are problems, Perezgonzales noted, if probabilities are treated as error rates: effect sizes are calculated, confidence intervals reported, and power is calculated all from a small set or even a singular exploratory study. All of these practices can only be justified on the bases of positing a stable reference distribution and cannot be justified on the basis of a "fiduciary" or a benchmark reference distribution. Brand, Bradley, Best, and Stoica, (2008) reported that exaggerated results may be published. Improbable significant findings represent some of the literature, whereas the unpublished failures comprise the now famous/infamous "file drawer" (Rosenthal, 1979).

Publishing practice in psychology as recommended by American Psychological Association (APA) manuals from the 1940s until the 1990ties directed researchers to indicate significance at the $.05, .01$, or .001 level without discussing whether these values were error rates or probabilities. Following Fisher's probability model a researcher would report an exact probability with the implication that smaller probabilities are better. Fisher was not stating that he "knew" all the characteristics of the reference distribution. Rather the sample was merely a sample that more or less approximated the population distribution, and, as such, it would be inappropriate to calculate an effect size if the inferential test is underpowered and involves a "fiduciary" or "convenient benchmark" distribution. Following the practice of reporting effect sizes in such circumstances, results in unstable (imprecise) estimates that are likely to be exaggerations (inaccurate) in the published literature.

The APA manual (2010) suggests reporting Confidence Intervals (CIs). CI calculations involve an inferential approach since the calculation of $C I s$ is based on the standard error. Thus 
NHST and CIs depend upon the sample size in any particular area, and since sample sizes can vary, it is analogous to using an elastic band as a ruler. Thus, significance comes and goes, and worse, in some areas the only calculations of effect sizes may come from misestimates of mean differences, variability, or a combination of both. An additional problem is that confidence intervals are often huge and exceed the magnitude of the effect size they are meant to bracket (Brand and Bradley, 2016). This results in a peculiar problem. If replication involves obtaining a value less than alpha, it is difficult to obtain results. If replication involves fitting in the confidence interval, it is difficult to fail to replicate (Brand \& Bradley, 2016).

The Fisher, Neyman and Pearson controversy can be portrayed as part of a larger dimension of probability testing. This dimension includes classical probability, well defined distributions, and exploratory science. Between exploratory science and well defined distributions there is a transition zone where shared characteristics of both exploration and knowledge co-exist. The dimension is multifaceted ranging from substantial but not perfect certainty to great uncertainty; deductive reasoning to inductive reasoning; described probabilities to estimated probabilities. Zone 1, or the approximate certainty end of the dimension, is characterized by classical probability; Zone 2 is best related to technological processes; Zone 3 is a middle ground with some but not complete knowledge; and Zone 4 involves exploratory science. Zone 3 follows Hulbert and Lombardi (2009). In this middle ground, researchers have some knowledge but they do not know the exact size of an effect or whether they have an adequately sized population to test an effect. In such cases, a researcher could allow a more liberal $\mathrm{p}$ value to continue work or to entice others to contribute more data. Bradley and Brand (2013) published a set of tables with small, medium and large effects, varying levels of power, and varying $N s$ to show the alpha level that is expected given those parameters. If the theory is 
dealing primarily with an established effect, but there are other potential effects of interest, then more liberal alphas levels could allow the entry of this less conclusive but possibly important subsidiary data into a field. We should point that other fields are working along similar lines. Mudge, Baker, Edge, and Houlahan (2012) combined Type 1 and Type 2 error rates to arrive at an optimal alpha both retrospectively for completed studies and prospectively for "to be conducted" work. If either or both of these approaches become widely accepted, they will reduce the volume of studies in the "file drawer" and increase the availability of more accurate effect size estimates. In addition, it can be argued that both Fisher and Neyman and Pearson had a nascent appreciation of this middle ground we call Zone 3. Neyman and Pearson clearly indicated that at times avoiding a type 2 error was more important than guarding against a type 1 error. Fisher suggested that "sometimes" depending on the subject and context a more liberal alpha probability indicating a difference was acceptable.

With a dimension and zones a user of inferential statistics can identify which zone they are working in. If you can control production processes to the point that you control even the materials fed into the process, you are working in the Neyman-Pearson area or Zone 2. You may design such a stable production process that a criteria set by a particular number of errors may obviate the need for statistical testing. This is because the characteristics of the reference distribution are controlled entirely by the process. For deductive reasoning, an error-based decision is valuable in this context. If the quality assessment is within a certain range, then it is reasonable to continue on producing. If it is out of the range, the "behavior' would be to shut production down with a $5 \%$ chance of error. Such decisions can only be achieved if the population distribution is thoroughly known, as it would be in a particular factory with a 
particular product. Fisher (1955, p 69) himself recognized the value of "acceptance" procedures reflecting upon his air travel, but he rejected these same procedures for science.

If you don't have much knowledge or only a hypothesis as to what the input or outcome of a study will be you are at the Fisher extreme or Zone 4. The key is that not only do you not control the population distribution you do not even have much knowledge about its characteristics. As such, you can take samples from it, and these samples, of course, are part of the larger population, but it is not known which part. These samples, of course, converge on an actual population distribution, but they only represent that distribution in a probabilistic way. Thus, the local population distribution is likely the one that is convenient but not necessarily universal. The uncertainty about distributional qualities allows the conclusion that a manipulation alters a sample so that it no longer looks like the unmanipulated distribution. It does not specify the difference in terms of the universal population distribution which may not exist.

A researcher may to move into middle ground or Zone 3 after several experiments, but that happens only after enough attempts to estimate parameters of a distribution are conducted. Once a researcher has gained enough knowledge to move into the middle zone, judgments about distributional characteristics should be made with more accuracy.

From our understanding, neither Fisher's or Neyman and Pearson's approach fit well into Zone 3. Fisher did, as mentioned, suggest a liberal $p$ is possible to accept for investigation, but in the main with a multitude of potential hypotheses, his interest at the time was in pursuing something that was likely obvious in a probabilistic sense. We had placed Neyman and Pearson firmly in Zone 2 with the specification of a distribution gained through control and verified through reiterated samples. This procedure allows the estimation of probabilities of Type 1 and 
Type 2 errors for making decisions about a sample, but as we argued earlier Neyman and Pearson encroach upon Zone 3 with the recognition that for errors sometimes "... it will be more important to avoid the first, in others the second" (Neyman and Pearson, 1933, p 296). In general, however, they made strong statements that Type 1 errors were more problematic “...an error of the first kind is more important to avoid than an error of the second kind..." (Neyman, 1950, p 265).

Theory and measurement are primary. An inference (the use of inferential statistics on measured values) about measurement is secondary. That inference may, in the absence of other information, signal something about the probability of the results, but replication with the admission of all legitimate attempts to replicate is the most reasonable way to establish and understand a finding. However, we also accept that inferential statistics can facilitate judgment if used properly. The dimension of "zones" to portray a model that guides researchers towards the correct application of inferential statistics.

The diagram represents four zones along a dimension of certainty, where certainty refers to the degree to which the inferential evaluative situation is fully defined. The zones are not mutually exclusive and it would take some experience or knowledge of an area to make some distinctions at the borderlines.

Figure 1 about here 
Zone 1 consists of classic probability. Essentially the data is categorical and certainty is virtually assured in the long run. There is, of course, some uncertainty related to improbable outcomes until a final outcome is achieved.

Zone 2 consists of Neyman and Pearson's attempt to create a situation in which virtually everything about the reference distribution is known. Production is perhaps the easiest example since virtually every variable such as the input materials, the length of the process, the temperatures, and the skill of the labor is controlled. Depending on the complexity of the process, inferential tests at various levels in the process reveal problems that may arise. The complexity of potential questions is substantial. As mentioned, Fisher said inferential statistics are the most primitive form of measurement. As more is known about the area more sophisticated forms of measurement can be brought to bear. He argued that for production lines Neyman and Pearson were following an appropriate model, but, as we agree, that model creates problems for the conduct of science (Fisher, 1955, p 69).

Zone 3 will be discussed after zone 4 because some of the conceptual orientation comes from zone 4.

Fisher described and designed statistics for Zone 4. He wanted to make a rough and ready distinction between those areas that were worth pursuing and those which were more ambiguous. Fisher developed his ideas prior to modern calculators and computers, where analyses were laborious. Allocating his time to various problems was a real decision. He studied wheat, which could vary each year depending upon wind, rain, sun and temperature as well as soil and disease infestations. Thus, his reference distribution varied, and the question is: did the comparison distribution also vary enough from the reference to suggest that one type of wheat was 
superior/inferior to another? On the one hand, he had to make a decision so he had in mind some criteria such as $p<.05$ or less, but, on the other hand, there could be circumstances in any given year that would allow a more liberal cutoff such as $p<.10$. He advocated the use of judgment in making these decisions. A researcher does not have an idea until the data are collected of the form of the distributions and the measure of central tendency. Ceiling or floor effects reflected in skewness could be operative and be taken into account by a researcher. His idea of reporting exact $p$ values reflected an attempt to gauge precision. Hubbard and Bayyarri (2003) point out that Fisher had a conception of the true population distribution of which he only had a sample, and then the question becomes whether the manipulated distribution was compatible or not with that null. The more the samples differ the greater the probability they will differ again even in a new context, and over time with consistent low $p$ values a fact might be established.

Zone 3 emphasizes the use of judgment depending upon what one is studying. An estimate of harm potential has been a factor in clinical drug tests. For example, Bendtsen, Bigal, Cerbo, Diener, Holroyd, Lampl, Mitsikostas, Steiner and felt-Hansen (2009) suggest a great many factors from drug interactions, functional impairment, to toxicity that need to be monitored and reported numerically and possibly a liberal $p$ value to be alert for harm. If undiscovered in initial testing, they may become obvious in larger clinical samples. If a more liberal $p$ value pertained in the initial tests, a researcher could have an awareness of potential side effects.

Fisher argued that his samples were only a sample of the possible/hypothetical population. His particular wheat fields in England belong to a limited population of wheat fields. He wanted his data to generalize to wheat all over England. A substantial difference between his reference sample and his tested sample, reflected in a lower $p$ value, is more likely to generalize. 
From the perspective of pre-computer or early computational aids and a limited number of statistical tests, it is reasonable to look for phenomena that have substantial effects. Fisher recognized the unknown nature of his actual population.

Neyman and Pearson (1933) were correct for recognizing that Fisher's procedures could, both in theory and empirically in very limited situations, model a situation in which the distance between means are described not only with very little chance of error but almost with exact specification of potential error. They also are correct since quality testing had long ago emerged (Student, 1908), for the clear applicability of their error decision model.

The current application of inferential statistics involve a hybrid model of NHST practice (Gigerenzer, 1993). Computing power and a massive numbers of research projects (Bradley \& Gupta, 1997) using inferential statistics in what Gigerenzer termed a "ritualistic" manner have created problems for psychology. We have no evidence that Fisher in setting low $p$ values anticipated 125,000 Psychologists in North America, plus some large number of Psychologists around the world who also have graduate and honors students, plus professors of Business, Biology, Education, Pharmacology, Nursing, etc, plus all of their students. Brand, Bradley, Best and Stoica (2008) showed with a small effect size tested with typical sample size would be significant yielding a Type 1 error once in seven tests and thus have an opportunity for publication. For every million researchers, roughly 140,000 papers of dubious value could be generated, and with a 10 to $20 \%$ percent acceptance rate as many as 28,000 papers could show up across the various disciplines. Our scenario only portrays a limited sample of the potential magnitude of the problem. Add to these erroneous findings as Gigerenzer points out, a feeling of confidence emanating from abuse of the Neyman and Pearson model. That is with power 
calculations based on previous exaggerated estimates, a statistically rare (Type 1) phenomena seems supported. The illusion of a supportable finding occurs because of 'the file drawer effect and the number of industrious participants most of whom file away their non-significant results while the fewer Type 1errors are published. A researcher, reader and editor could have false confidence in an apparently low probability event with seemingly adequate power to find it.

It is unfortunate that neither Fisher or Neyman and Pearson could anticipate the popularity of their methods and the mechanistic ways of application. Fisher had mapped clearly two of our zones and would clearly support the use of Neyman and Pearson methods in Zone 2 while insisting on the application of his approach in Zone 4. Neyman and Pearson definitely were successful in defining Zone 2 and showed signs of thinking about Zone 3 with a more liberal alpha when power was not optimal. Both Fisher and Neyman and Pearson have been victims of their own success. 


\section{References}

American Psychological Association. (1999). Publication manual of the American Psychological Association (4 ${ }^{\text {th }}$ ed.). Washington, DC: Author.

American Psychological Association. (2002). Publication manual of the American Psychological Association (5 ${ }^{\text {th }}$ ed.). Washington, DC: Author.

American Psychological Association. (2010). Publication manual of the American Psychological Association ( $6^{\text {th }}$ ed.). Washington, DC: Author.

Babbie, E. R. (2001). The practice of social research. (9 $9^{\text {th }}$ ed.) Wadsworth: Belmont Ca.

Bakan, D. (1966). The test of significance in psychological research. Psychological Bulletin, $66,423-437$.

Bendtsen, L., Bigal, M. E., Cerbo, R., Diener, H. C., Holroyd, K., Lampl, C., Mitsikostas, D.D., Steiner, T.J. \& Tfelt-Hansen, P. (2009) Guidelines for controlled trials of drugs in tension-type headache: Second edition. Cephalalgia 30(1) 1-16

Box, J. F. (1978). R. A. Fisher: the life of a scientist. John Wiley

Bradley, M. T. \& Brand, A. (2013). Alpha values as a function of sample size, effect size, and power: accuracy over inference. Psychological Reports: Measures \& Statistics, 112, 3, 110.

Bradley, M.T. \& Brand, A. (2016). Accuracy when inferential statistics are used as measurement tools. BMC Research Notes20169:241. DOI: 10.1186/s13104-016-2045-Z

Bradley, M. T., Brand, A., \& MacNeill, A. L. (2012). Interpreting effect size estimates through graphic analysis of raw data distributions. In Cox, P. Rodgers, P. \& Plimmer, B. (Eds) Diagrams. 117-123. Springer-Verlag: Berlin. 
Bradley, M. T., Brand A., \& MacNeill, A. L. (2013, June). Effect size reporting reveals the weakness Fisher believed inherent in the Neyman-Pearson approach to statistical analysis. Poster presented at the annual meeting of the Canadian Psychological Association. Quebec City, Quebec

Bradley, M. T. \& Gupta, R. D. (1997). Estimating the effect of the file drawer problem in metaanalysis. Perceptual \& Motor Skills, 85, 719-722.

Bradley, M. T. \& Stoica, G. (2004). Diagnosing estimate distortion due to significance testing in literature on the detection of deception. Perceptual and Motor Skills, 98(3), 827-839. doi: 10.2466/Pms.98.3.827-839

Branch, M. (2014). Malignant side effects of null-hypothesis significance testing. Theory \& Psychology, 24, 256-277.

Brand, A., Bradley, M. T., Best, L., \& Stoica, G. (2008) Accuracy of effect size estimates from published psychological research. Perceptual \& Motor Skills, 106, 645- 649.

Brand, A., \& Bradley, M. T. (2016) The Precision of Effect Size Estimation From Published Psychological Research Surveying Confidence Intervals. Psychological Reports 118, 1 154-170. doi: 10.1177/0033294115625265

Cohen, J. (1994). The earth is round ( $p<.05)$. American Psychologist, 49, 997-1003.

Cowles, M. \& Davis, C. (1982). On the Origins of the .05 Level of Statistical Significance. American Psychologist. 37, 5, 553-558

David, H. A. and Edwards, A. W. F. (2001). Annotated Readings in the History of Statistics. Springer, New York.

Fisher, R. A. (1922). On the Mathematical Foundations of Theoretical Statistics. Philosophical 
Transactions of the Royal Society of London 222, 309-368.

Fisher, R. A. (1925). Statistical methods for research workers. Oliver \& Boyd: Edinburgh.

Fisher, R. A. (1926). The arrangement of field experiments. Journal of the Ministry of Agriculture, 33, 503-513.

Fisher, R. A. (1935). Statistical Tests. Nature, 136, 474.

Fisher, R. A. (1942). The design of experiments. ( $3^{\text {rd }}$ ed.) Oliver \& Boyd: London.

Fisher, R. A. (1955). Statistical methods and scientific induction. Journal of the Royal Statistical Society. Series B (Methodological) 17, 1, 69-78.

Fisher, R. A. (1973). Statistical methods and scientific inference. ( $3^{\text {rd }}$ ed.) Hafner Press: New York.

Gigerenzer, G. (1993). The superego, the ego, and the id in statistical reasoning. In G. Keren, \& Lewis. (Eds.), A Handbookfor Data Analysis in the Behavioral Sciences:Methodological Issues (pp. 311-339). Hillsdale, New York: Lawrence Erlbaum Associates.

Halprin, P. F. \& Stam, H. J. (2006). Inductive inference or inductive behavior: Fisher and Neyman-Person approaches to statistical testing in psychological research (1940-1960). The American Journal of Psychology, 119, 625-653.

Hubbard, R. \& Bayarri, M. J. (2003). Confusion over measures of evidence (p’s) versus errors (a) in classical statistical testing. The American Statistician, 57, 171-182. 
Hurlbert, S. H. \& Lombardi, C. M. (2009). Final collapse of the Neyman-Pearson decision theoretic framework and the rise of the neoFisherian. Annales Zoologic Fennici, 46, 311349.

H.A. Keuzenknmp, H. A, \& Magnus, J.R. (1995) Journal of Econometrics 67, 5-24.

Kline, R. B. (2013). Beyond significance testing: Statistics reform in the behavioral sciences (2nd ed.). Washington, DC: American Psychological Association.

Lenhard, J. (2006). Models and Statistical Inference: The Controversy Between Fisher and Neyman-Pearson. British Journal for the Philosophy of Science 57 (1):69-91. doi: 10.1093/bjps/axi152

Mudge, J. F., Baker, L. F. Edge, C. B., Houlahan, J. E. (2012). Setting an optimal $\alpha$ that minimizes errors in null hypothesis significance tests. PLoS One;7:e32734

Neyman, J. \& Pearson, E. S. (1933). On the Problem of the Most Efficient Tests of Statistical Hypotheses. Philosophical Transactions of the Royal Society A: Mathematical, Physical and Engineering Sciences 231 (694-706): 289-337. doi:10.1098/rsta.1933.0009

Neyman, J. (1937). Outline of a theory of statistical estimation based on the classical theory of probability. Philosophical Transactions of the Royal Society A 236: 333-380.

Neyman, J. (1950). First Course in Probability and Statistics. New York: Henry Holt.

Perezgonzales, J. D. (2014) A reconceptualization of significance testing. Theory \& Psychology, 24: 852-859, doi:10.1177/0959354314546157

Rosenthal, R. (1979). "The "File Drawer Problem" and the Tolerance for Null Results", Psychological Bulletin 86 (3): 638-641, doi:10.1037/0033-2909.86.3.638 
Salsburg, D. (2001) The Lady Tasting Tea: How Statistics Revolutionized Science In The Twentieth Century Henry Holt and Co., New York.

Sharp, D. \& Poets, S. (2015, June) Where Are the Boys? Statistical Implications of the Absence Of Men from Psychology Participant Pools. Presented at the annual meeting of the Canadian Psychological Association. Ottawa, Ontario.

Sterling, T. D. (1959), Publication decision and the possible effects drawn from tests of significance - or Vice Versa. Journal of the American Statistical Association, 54, 30-34.

Stevens, S. S. (1946) On the theory of scales of measurement Science 103 (2684): 677680. doi:10.1126/science.

Stigler, S. M. (1986) The history of statistics: The measurement of uncertainty before 1900 Belknap Press, London England.

'Student' (1908). The probable error of a mean. Biometrica, 6, 1-25.

Tukey, J. W. (1977). Exploratory data analysis. Reading, Mass.: Addison-Wesley

Wald and Abraham (1945). Sequential Tests of Statistical Hypotheses. Annals of Mathematical Statistics. 16 (2): 117-186. doi:10.1214/aoms/1177731118

Wasserstein, R. L. \& Lazar, N. A. (2016): The ASA's statement on p-values: context, process, and purpose, The American Statistician, DOI: 10.1080/00031305.2016.1154108 


\begin{tabular}{|l|}
\hline The odds are \\
known and \\
with the right \\
process will \\
reflect those \\
odds
\end{tabular}

Studied distributions that have been sampled and resampled such that error rates are established

Neo-Fisher
with graded
probability
cutoffs
depending on
the
experimental
context.

Basic science: only samples are known and those samples estimate the not exactly known population parameters. 